BENJAMIN OLTRA

\title{
GRUPOS DE INTERES E INTELECTUALES EN DAHRENDORF
}

Es concebible, quizás plausible incluso, que la presencia de estructuras de poder y subordinación en las sociedades humanas sea la razón última de la presencia de protestas $y^{\prime}$ resistencias, $o$, desde un punto de vista más: general aún, de la oposición y el desorden.

(Dahrendorf, 1967).

Una visión general de las obras de Dahrendrof permite constatar que son dos las cuestiones que le importan. La primera es una cuestión en sí ética. Trata de ser un sociólogo «engagé» inspirado por la «fibra motal' de sus predecesores» (1968: 123). La segunda cuestión, ésta propiamente científica, consiste en llevar a cabo una tevisión crútica de Marx y de Parsons a fin de construir una teoría de la sociedad que se base en una teoría del choque de intereses y que presente una sintesis más nueva y «realista». Sobre la base de este esquema, este artículo trata de exponer un aspecto de la obra de Dahtendorf: la articulación social de los grupos de interés, las élites, y la función de los intelectuales. Respecto a sus explicaciones en torno a los intelectuales, estamos de acuerdo con la 
crítica que señala que las conclusiones de Dahrendorf no son coherentes con sus puntos de partida teóricos, y que asimismo indica que cae en un cierto reduccionismo. Sin embargo, Dahrendorf ofrece un esquema sugerente para un estudio más detallado de los intelectuales, que aún está por hacer.

Causas del conflicto entre las clases

El análisis que de los grupos de interés hace Dahrendorf trata de demostrar que la naturaleza coercitiva de la autoridad es la variable independiente que explica los choques entre los diversos intereses funcionales. Estos intereses, dadas ciertas condiciones, llevan a la formación de grupos organizados de interés a partir de organizaciones sociales limitadas ("quasigrupos»). Dahrendorf trata aquí de llegar a una síntesis entre la teoría de las clases y los intereses de las clases de Marx y la metodología de la teoría de la acción de Parsons. ${ }^{1} \mathrm{La}$ síntesis crítica de ambas teolías es presentada en su obra Class and Class Conflict in Industrial Society (Clase y conflicto de clases en la sociedad industrial) (1959).

Dahrendorf empieza explicando la principal herramienta analítica de su teoria: la autoridad en Ias «asociaciones imperativamente coordinadas» (a.i.ci) (1959: 165-67). Subraya la distinción que hacía Weber entre poder y autoridad, en la que la autoridad se relaciona siempre con las posiciones o papeles sociales. Se muestra en completo acuerdo con Weber en la defi-

1. Según Marx la Historia avanza debido a la lucha de clases. Esto se debe a que existe una dialéctica de la «estructura económica» de las sociedades que lleva a que se establezcan grandes diferencias en la propiedad de los medios de producción. Los intereses de las clases son explicados en éstos términos. Es más, para Marx la lucha de clases es una lucha política. La estructura económica (subestructura) está en contradicción con las instituciones del poder y la autoridad (superestructura) en toda fase histórica. El poder es, por to tanto, el instrumento de una clase dominante (como la burguesía) para conservar y reproducir sus intereses de clase y optimir a la otra clase (el proletariado). Por otro lado, la teoría funcional estructural de Parsons explica el orden social del proceso de internalización social de normas y valores. Et orden social está basado en el consenso. Pero la realización del consenso requiere ciertos instrumentos. Uno de ellos es la autoridad (elemento estático) que es el control que el sistema sacial requiere para lograr cumplir con su función de integrador de normas, valores y objetivos sociales. El otro es el poder (elemento dinámico) que es la «capacidad de movilizar los recursos de la sociedad pata la consecución de objetivos» (Parsons, 1960: 221). 
nición que de la autoridad da éste: «la probabilidad de que una orden con un contenido específico sea obedecida por un grupo de personas dado" (Weber, 1959: 53). ${ }^{2}$ Esta base teórica (a.i.c.) implica tres nociones importantes: a) autoridad (o dominio), b) coetción, y c) conflicto. Dabrendorf propone esas tres nociones como alternativa a la triple base conceptual utilizada por Parsons en su análisis del sistema social, a saber: a) valores, b) asociaciones voluntarias, y c) consenso.

Veamos qué significa «autoridad» para Dahrendorf. Afirma que la autoridad es un elemento estructural universal, y así que «hay telaciones de autoridad en donde quieta que haya personas cuyas acciones estén sometidas a prescripciones legítimas y sancionadas que se originan fuera de ellas pero en el seno de estructuras sociales» (1959: 168); y esto ocurre en todas las sociedades. ${ }^{3}$ Sin embargo, tal aspecto de la autoridad sólo parece relevante en un modelo de sociedad coetcitiva. Dahrendorf sugiere estas dos posibilidades de relaciones: a) La distribución de autoridad en las a.i.c. es la causa última que explica el conflicto entre grupos; b) la distribución de la autoridad es la variable independiente que explica la formación de dos (y sólo dos) grupos sociales: «soberanos» y «súbditos».

2. Según esta noción, autoridad significa tanto «dominio como «sometimiento. Dahrendorf añade: «En el análișis del conflicto nos interesa inter alia la generación de los grupos conflictivos por las relaciones de autoridad an la asociaciones imperativamente coordinadas" (1959: 167).

3. Dahrendorf se refiere aquí al «aspecto feo» de la autoridad en tos mismos términos que los utilizados por Parsons en su concepto del poder como suma-cero. Según Dahtendorf, Parsons quiere decir que si se contemplan las funciones destructivas de la autoridad whay dos grupos o conjuntos de personas, uno de los cuaies tiene la autotidades tanto en cuanto el otro catece de ella» (Dahrendorf, 1959: 170). Así, para Dahrendorf hay siempre una dicotomía de posiciones en la a.i.c. respecto a la posesión de la autoridad. Sin embargo Dahrendrof no está de acuerdo con Parsons en la crítica que éste hace de Mills. Parsons compara la distriburción de la autoridad con la distribución de la riqueza en las sociedades. Para Dahrendorf, tal comparación es un malentendido. El considera que la riqueza y los indicadores de estratíficación social no pueden concebirse como conceptos surna-cero (en ésto está de acuerdo con Parsons). Pero, según Dahrendorf, la autoridad no permite la creación de escalas cuantitativas; asi, la autoridad es una realidad que suma cero y las «llamadas jerarquías de autotidad... son de hecho jerarquías dentro del lado «más» de la autoridad... pero hay en toda asociación un lado «menos» que consiste en aquéllos que están sometidos a la autoridad en lugar de participar en su ejeccicios (Dahrendorf, 1959: 170.171). 
Grupos de interés e intelectuales en Dahrendorf

\section{Grupos de interés}

Al paso que presenta su noción de autoridad Dahrendorf crea el concepto de «interés» con el fin de explicar los orígenes y la dinámica de los grupos, o sea, cómo los «quasi-grupos» se convierten en «grupos de intetés». ${ }^{4}$ Dahrendorf propone el concepto de «interés», opuesto al de «valo. tes» en Parsons, en el sentido de «orientación de la acción de los ocupantes de posiciones definidas» (1959: 175). Dahrendorf adopta el concepto de interés de Marx, con sus orientaciones generadas estructuralmente, o bien sigue una noción no-psicológica de interés. Hay, por lo tanto, intereses cuando los dos grupos de las a.i.c. tienden hacia el mantenimiento o la modificación de un status quo. Esto supone que en toda a.i.c. estos dos tiv pos de interés se encuentran en conflicto. Siguiendo la idea de Weber según la cual la conservación o cambio de las estructutas de autoridad puede expresarse basándose en su legitimidad, Dahrendorf asume que: ${ }^{5}$

La legitimidad de la autoridad debe ser siempre precaria. Hay siempre un conjunto de posiciones y quienes las ocupan que representa la duda institucionalizada en la legitimidad del status quo de la distribución de la autoridad.

En este sentido, la afirmación de la existencia de intereses «objetivos» en favor del cambio de cualquier estructura de autoridad dada, puede expresarse también en términos de la potencial ilegitimidad de todas las relaciones de autoridad. (1959: 176).

En el contexto de una teoría de la coerción, los papeles contribuyen más a la existencia de conffictos que a la de integración. Para reemplazar

4. Dahrendorf anaizza el proceso de los grupos conflictivos refiriéndose a un modelo cuyo punto de partida básico es que «los grupos conflictivos se basan en la distribución dicotomizada de la autoridad en las asociaciones imperativamente coordinadas... [y] las diversas posiciones de autoridad en las asociaciones implican, para quienes las ocupan, intereses en conflictop (1959: 174).

5. Es importante tener en cuenta que Dahrendorf afirma también que la categoría analítica de «interés» en la teoría de la coerción debería ser entendida en estricta analogia con la de la esperanza de conseguir papeles. Esto supone que los intereses objetivos son intereses en relación a un papel, «la orientación de la conducta de esperar en relación con los papeles de la autoridad en una asociación imperativamente coordinadas (1959: 178) (sic). 
los problemas típicos de la teotía de los papeles, Dahtendorf propone los conceptos de interés latente e interés manifiesto. Son intereses latentes las «orientaciones del comportamiento inherentes a las posiciones sociales, sean o no conscientes de ello quienes las ocupan... y que contraponen dos conjuntos de posiciones en toda asociación imperativamente coordinadas (Dah* rendorf, 1959: 237). Por otro lado, son intereses manifiestos las «orien. taciones del comportamiento articuladas y conscientes de individuos y que contraponen colectividades de individuos en toda asociación imperativamente coordinadas (Dahrendorf, 1959: 238). ${ }^{6}$ Dahrendorf utiliza ambos conceptos para explicar el proceso de generación de grupos de interés organizados, así como su papel en el conflicto entre las clases.

Al desarrollax el tema de los quasi-grupos y grupos de interés, Dahrendorf construye una teoría que trata de explicar cómo emergen los conflictos entre los diversos grupos, y qué tipo de conjuntos se forman. Estos últimos son conceptualizados sobre la base del sustrato de hecho de la sociedad, y no en términos de fenómenos normativos. Siguiendo las ideas de Ginsberg, utiliza el término de quasi-grupo en el sentido de: conjuntos con intereses de papel latente similares, los cuales potencialmente tienden tanto a cambiar el status quo como a mantenerlo. Por definicion, estos quasi-grupos no están bien organizados (1959: 238). La principal característica de estos conjuntos es la presencia de sus intereses latentes comunes que, per se no deben ser interpretados simplemente como fenómenos psicológicos. Los quasi-grupos, sin llegar a ser organizaciones, son «campos de teclutamiento» para los grupos de interés. Los grupos de interés son «cualquier colectividad organizada de individuos que comparten intereses manifiestos» (Dahrendorf, 1959: 239), y son tanto grupos como factores de conflictos entre grupos. Tienen estructura, organización, partidarios y valores explícitos e ideologías.

6. Aquí muestra Dahrendorf su típico exlecticismo teduccionista, al sugerir que el «interés manifiesto» significa casi lo mismo que la «conciencia de clase» en Matx. Por otro lado, establece analogías entre el interés manifiesto y las «esperanzas de obtener papeles» de Parsons, mientras considera que él no está utilizando las características filosóficas ni especulativas del concepto de Marx por una parte, ni el partidismo conservador de Parsons por otra, Podríamos poner en duda si es posible trabajar con tales simplificaciones excesivas y, al mismo tiempo, presentar la interpretación que se da como una teoría avanzada.

7. Para Dahrendorf las diferencias específicas entu'e quasi-grupos y grupos de interés se hacen mayores «por su origen en la estructuta de autoridad de las asociaciones o... por las características formales de sus inte:teses (latentes o manifiestos) subyacentes como intereses relacionados con la legitimidata de las relaciones de dorainio y sometimiento" (1950: 181). 
Dahrendorf subraya los ptoblemas «empíricos» de los grupos de interés, expresados en la formulación de estas preguntas: ¿Cuáles son las conexiones entre los quasi-grupos y los grupos de interés? ¿Cuáles son las fases intermedias en la evolución de los quasi-grupos y los grupos de interés? ¿Qué tipos de variables específicas hacen su transformación posible?

Hasta aquí se refiere principalmente a categorías, pero a partir de este punto trata de explicar las líneas que conectan los quasi-grupos a los grupos de interés, utilizando como ejemplo El Dieciocbo de Brumario de Luis Bonaparte (1852) ${ }^{8}$ Los grupos se convierten en grupos de interés «en condiciones ideales" ya que:

En toda asociación imperativamente coordinada, pueden distinguirse dos quasi-grupos unidos por un interés latente común. Las orientaciones de su interés están determinadas por la posesión o exclusión de la autoridad. A partir de esos quasi-grupos se reclutan los grupos de interés, cuyos programas atticulados defienden o atacan la legitimidad de las estructuras de autoridad vigentes. (Dahrendorf, 1959: 183-84).

Dahrendorf esboza las tres series de condiciones empíricas bajo las que se organizan los gtupos de interés: ${ }^{9}$ a) condiciones técnicas (miembros, personal y estatutos); b) condiciones políticas (libertad de coalición); y c) condiciones sociales (comunicación y reclutamiento organizado). Para Dahrendorf «la formación de grupos de interés organizados es posible sólo si el reclutamiento de los quasi-grupos sigue un esquema estructural y no el azar» (1959: 187$)^{10}$

8. Marx explica cómo Luis Bonaparte justificó sus pretensiones de asumit el poder refiriéndose a los intereses latentes de un quasi-grupo: los pequeños campesinos independientes, la clase más numerosa de Francia y típicamente carente de organización, ideología, etc. Luis Bonaparte y el interés manifiesto tepresentado por su clase crean su «farsa» (según el término utilizado por Marx), organizándose sobre la base de una «supuesta legitimidad» otorgada por los campesinos.

9. Dahrendorf relaciona las seis características relevantes para las instituciones (aquí los grupos de interés organizados) con los términos: estatuto, personal, normas, instrumentos materiales, actividades organizadas, y función objetiva.

10. ¿Qué papel juega la dinámica de los grupos de interés en el cambio social? Los problemas que se plantean como base aquí son: a) Si las a.ic. están sujetas a cambios radicales, hay pocas probabilidades de que los quasi-grupos puedan llegat a convertirse en grupos de interés (sociedades después de una revolución, por ejern- 
Elites

Dahrendorf no presenta una explicación sistemática del problema de las élites en el conflicto de clases. ${ }^{11}$ Sin embargo no está de acuerdo con el planteamiento general de Pareto, Mosca y Aron. Su punto de partida es determinar si es posible identificat, si hay características comunes a los «ocupantes de posiciones de dominio y sus grupos de interés, y quienes ocupan posiciones de sometimiento» (1959: 193). Pareto, Mosca y Aron explican la formación de clases y la estructura de grupos desde el punto de vista de los grupos que ocupan las posiciones de dominio, pero estos autores presuponen un modelo de dos clases y subtayan especialmente las estructuras de autoridad. Sin embargo Dahrendorf señala que su obra es simplemente un «origen aproximado de una teoría del conflicto» (1959: 194). ${ }^{\mathrm{t2}}$

Basándose en El Dieciocho de Brumario, explica Dahrendorf que los «grupos suprimidos» en tanto en cuanto son campos de reclutamiento para intereses manifiestos, pueden convertirse en grupos de interés; y ésto es especialmente claro en las sociedades industriales «porque en estas socie-

plo, la revolución Soviética). b) En su discusión de las reorías de Warmer, Centers, Hoggart, Popitz y Willener y otros que tratan el tema de la formación de grupos conflictivos al nivel de investigación psicológica Dahrendorf está de acuerdo en que ciertas condiciones psicológicas no estructurales (por ejemplo, la internalización del intetés por los papeles) puede ayudar a explicar el contlicto.

11. Es cierto que Dahrendorf realiza un análisis empírico de los «cambios en la élite alemana» en Sociedad y democracia en Alemutnia (1967: 217.296) así como un esbozo crítico en Clase y conflicto de clases. En ambas obras, a nuestro parecer, Dahrendorf no llega a establecer una relación satisfactoria entre los grupos de intexés y las élites, en el contexto de la teoría del. conflicto de clases.

12. Dahrendorf selecciona cinco clases de problemas: a) ¿Qué supone para la autoridad y el cambio el tamaño de los grupos dominantes? Dahrendorf supone que nunca se ha discutido la tesis de que las élites (dominanies) son pequeñas en relación a su equivalente dominado. Pero este supuesto debe ser corregido si lo que se consideran son las sociedades industriales porque «la delegación de autoridad en la industria, el estado y otras asociaciones hace posible que en las sociedades indus. triales los grupos dominantes ya no sean pequeñas minotías sino que en tamaño casi correspondan al de los grupos dominados» (Dahrendorf, 1959; 195). Según esta hipótesis, las tesis de Pareto, de Mosca y de Aron deben ser corregidas. Esto se debe a que hay considerables grados de autoridad y a que el poder puede estar relativamente distribuido. b) para Dahrendorf, las diversas «propiedades» encontradas por Pareto y Mosca en los grupos dominantes por lo que se refiere a la defensa de st 
dades deja de existir uno de los obstáculos a la organización de grupos de sometidos que caracterizaba sociedades anteriores: la imposibilidad de comunicación» (Dahrendorf, 1959: 200). Al nivel de análisis empírico, Dahrendorf aplica indiscriminadamente los conceptos de grupos de interés y élites (siempre en relación al conflicto) al estudio de Alemania. ${ }^{13}$ En Society and Democracy in Germany (1967) (Sociedad y democracia en Alemania) esboza las cuatro condiciones sociales necesarias para una democracia liberal y trata de mostrar las razones que explican su fracaso histórico. ${ }^{14}$ Respecto las élites, la condición que hace que una democracia sea libetal depende en parte de hasta qué punto esas élites «reflejen la gama de colores y diversidad de intereses sociales» 1967: 274).

En Clase y conflicto de clases en la sociedad industrial (1959) Dahrendorf afirma que la clase dominante (la élite clave de un grupo de interés) consiste en dos constantes históricas (burocracia y gobiemo), y una variable (el grupo del veto incorporado a la política gubernamental). Este esquema es aplicado a su análisis de los cambios estructurales de la élite alemana, y permite distinguir dos niveles en el seno de la élite del poder: posición social, y actitud política. En el primero se encuentran las «élites estable-

pasición en el poder, son análisis meramente pre-sociológicos. c) Dahrendorf rechaza la idea de la existencia de esuperioridad» concreta en la organización de los grupos dominantes (en relación a los dominados) debido a la existencia de una «cultura común" a ambos. d) Mosca y Pareto reducen ei concepto de clase dominante a Ia esfera del poder y el dominio político. Aron, por el contratio, distingue entre poder político y poder económico con lo cual presupone una superposición de dos clases dominantes. Para Dahrendorf, ninguno de ellos relaciona autoridad con el concepto de a.⿱亠乂.c. de modo que la expresión «clase dominante» tiende a confundir más que a aclarar ya que no incluye la posibilidad de que existan grupos dominantes en conflicto, competencia o coexistencia. e) Critica la identificación del estrato superior de la sociedad (grupos dominantes) con los grupos conflictivos dominantes, ya que esto supone que los grupos dominantes determinan todo el «nivel de cultura» $y$ «cambio» en Ia sociedad.

13. A Dahrendorf le interesa no solamente el estudio del conflicto hecho desde un punto de vista abstracto, sino también en concreto, como en el caso de fenómenos como: la democracia liberal (1967 b), el problema de la libertad $(1961,1963)$, y la aparición de contrarréplicas (como la aparición del modelo de Dictadura Nazi) en este contexto (1967 b).

14. Dahrendorf distingue también entre: a) el terreno de participación individual en el que es condición indispensable de una democtacia liberal un cierto grado de extensión del papel de la ciudadanía; b) el terreno en que se regula socialmente el conflicto; el grado de extension de la necesidad de regular racionalmente los conflictos; c) el terreno de los valores, en el que es condición del liberalismo un cierto grado de aceptación social de los valores públicos 1967: 274). 
cidas» si es alto el grado de su coherencia y mohesión interna; y las «élites abstractas», no como fenómeno real sino como fenómenos de orden, construcciones sociales de los científicos quasi-grupos). Desde el punto de vista de la actitud política, Dahrendorf distingue entre las élites políticamente uniformes, que son aquéllas cuya ideología política es unitaria y con intereses comunes de gran cohesión; y las élites políticamente multiformes (no plutalísticas), si aparecen como una diversidad tanto ideológicamente como en cuanto a sus intereses. La tipología consta de cuatro tipos ideales de élites del poder:

Los cuatro tipos ideales de élites del poder

\begin{tabular}{llc} 
Tipo social & $\begin{array}{c}\text { Actitud politica } \\
\text { Uniforme }\end{array}$ & Multiforme \\
\hline establecida & Elite autoritaria & Elite liberal \\
Abstracta & Elite totalitaria & $?$
\end{tabular}

La pregunta que sigue inmediatamente de este esquema es, ¿cómo pueden interpretarse los totalitarismos actuales y pasados en términos de élites abstractas o uniformes?

Weingart ha criticado el concepto de élite de Dahrendorf, y sugiere que «tiene un valor poco más que decorativo» (1969: 163). Hablando en general, el esquema de las condiciones sociales de la democracia que Dahrendorf traza no propotciona una explicación catusal para este complejo proceso de la sociedad industrial. Sus cuatro condiciones sociales necesatias para la democracia y la aparición de los totalitarismos modernos son útiles, de hecho, para explicar una tautología: no hay democracia en cuanto hay algo antidemocrático, y viceversa.. ${ }^{15}$

15. Desde el punto de vista de los grupos de inter:s y las élites esto es particularmente cierto. Weingart considera que ta tesis de Dahrendorf es una tesis ex post facto, que introduce un «cierto grado de determinisno» en el análisis» (Weingart, 1969: 164). Ver también Dahrendorf (1963: 209). 
Intelectuales: la metáfora del «bufón»

Dahrendorf ofrece ideas fructíferas en torno al discutido tema del intelectual. Trata este tema en tres de sus obras: en Clase y conflicto de clase en la sociedad industrial (1959), respecto a los nuevos grupos de interés y élites; en Sociedad y Democracia en Alemania (1967), a lo Largo de un análisis empírico de los intelectuales alemanes; y en su artículo "The Intellectual and Society: The Social Function of the «Fool» in the Twentieth Century» (ed. por Rieff, 1970: 53-56). (El intelectual y la sociedad: la función social del «bufón» en el siglo xx).

Parece que Dahrendorf trata de dar hipótesis para explicar dos importantes problemas: por un lado, equiénes son los intelectuales y cuál es su puesto en Ia jerarquía del orden social?; y, por otro, ¿cuáles son sus lazos con los quasi-grupos, grupos de interés, dominadores y dominados, y cuáles son sus papeles principales en relación al orden establecido, el conflicto y el cambio? Esbozamos estas dos series de problemas según la tipología general de las élites de Dahrendorf, que las clasifica de acuerdo con su tipo social y su actitud política.

Estamos particularmente interesados por los intelectuales y sus actitudes políticas como parte del análisis de la diversidad y uniformidad de las élites. (1967: 283).

Es bastante claro que a Dahrendorf no le interesa definir al intelectual, aunque muestra cierta preocupación por la necesidad de manejat una definición precisa. Por esta razón critica la idea de Russell del intelectual como tercer tipo, como «los que se apartan» (Russell, 1957). Es más, pone en duda sobre todo la definición de los intelectuales que da Lipset: "creadores» $\mathrm{y}$ «distribuidores» de cultura. ${ }^{16}$ Para Dahrendorf, ambas explicaciones no logran construir correctamente al intelectual porque sólo ven una de las características de su papel: la idea de que lo característico fundamental del intelectual es su posición y papel en el mundo simbólico del hombre (Dahrendorf, 1967: 281). Pero para Dahrendorf el papel del intelectual no

16. Lipset considera como intelectuales a «todos aquéllos que crean, distribuyen y aplican la cultura, es decir, el mundo simbólico del hombre en el que se incluyen el arte, la ciencia y la religión» (1960: 131). 
puede, normalmente, ser localizado en el seno de la estructura de las ocupaciones; hay que hallarlo más bien en el terreno del poder y la oposición (1967: 281). Esta idea setá más ampliamente desarrollada más abajo.

Respecto al lugar ocupado por el intelectual en la estructura de clase, Dahrendorf piensa que en las sociedades capitalistas, las intelligentsias son reclutadas sobre todo entre las ciases propietarias (1959: 80), en contraste con la postura de Nemchinov ${ }^{17}$ en su análisis de: la intelligentsia soviética. Sin embargo, para Dahrendorf la evolución de los intelectuales a través de la estructura social de las clases, sigue una dinámica compleja. Explica la posición y el papel del intelectual rechazando la conceptualización del intelectual como profesión o status. Atribuye el papel del intelectual a las estructuras políticas y de poder existentes, dando sobre todo importancia a su libertad y sus sujeciones, su papel sui generis, y dice que los intelec. tuales, en sí mismos, "simbolizan la estructura de su sociedad de una forma especial» (1967: 283). Explica la posición y el papel del intelectual utilizando la metáfora sociológica (impresionista) del «tonto» o el «bufón de la corte» (1970), también utilizada por Coser (1964) para explicar la función de cabeza de turco del conflicto social que tienen. Dahrendorf utiliza la metáfora del bufón en el contexto de sus ideas sobre el conflicto social (1958, 1959: 205), y también en el Homo Sociologicus (1968: 32). El plantea el problema de la forma siguiente:

Todos los que están en Ia parte superior, en medio o en la parte inferior de la sociedad desempeñan su papel social - pero el bufón se define porque siempre actúa de forma nada característica. Su papel es no interpretar papel alguno. EI bufón no está arriba pues no puede dictar a los demás las leyes de sus acciones. Tam. poco está debajo porque actúa como conciencia crítica de los poderosos, y se toma libertades que si fueran tomadas por «los que están abajò serían castigadas... el poder del bufón está en su libertad en relación con la jerarquía del orden social, es decir, que habla tanto desde fuera como desde dentro de ese orden... Pero

17. Dahrendorf discute la tesis de Nemchinov sobre la actual intelligentsia sovietica entendida como un estrato social intermedio reclutado en la clase obrera. Dahrendorf señala que Nenchinov incluye en su concepto de intelligentsia no s6lo los intelectuales entendidos desde un punto de vista clásico, sino también a los técnicos, obreros administrativos y los que ocupan las posiciones de autoridad. Dominan el Estado, la industria, la universidad, y así sucesivamente, así como sus respectivas burocracias. Para Dahrendorf la falacia de su punto de vista lleva a Nemchinov a caer en graves contradicciones. Dahrendorf analiza realidades muy diferentes con tesis marxistas, que en el último siglo han sido carnbiadas. 
los bufones de las sociedades modernas... son los intelectualestambién muy despreciados... Como bufones de la corte de la sociedad contemporánea, todos los intelectuales tienen la tarea de dudar de todo lo que es evidente, de hacer relativa toda autoridad, de preguntar aquelias preguntas que nadie se atreve a plantear. Desde luego, no son cuestiones cómodas" (1970: 54-55).

En nuestra opinión, hasta ahora la sociología del intelectual ha entendido su papel en los sentidos siguientes: a) el intelectual como «pouvoir spirituel» (Alfred Weber y en algunos aspectos el pensamiento de Manheim); b) el intelectual como epifenómeno subversivo (pensamiento conservador europeo: Burke, Maurras, etc., y algunas declaraciones de Aron, entre ellas la ideología del «fin de la ideología»); c) el intelectual como factor del cambio social revolucionario (Marx, Lenin, Marcuse, Mills entre otros; y d) el intelectual como un papel o serie de papeles diversos. Esta última línea de pensamiento sugiere una salida diferente tanto de la idea de que los intelectuales están en favor del orden establecido, como de la que ve su función como en favor del cambio social (algunos aspectos de Weber y Gramsci, y también Merton, Shils, Lipset, Huszar, Marsal, Coser y Kadushin entre otros). De esta tipología, nos parece, Dahrendorf tiende a seguir la línea auto-encomiástica (pouvoir spirituel) del intelectual aunque por otro lado, ve los problemas desde un punto de vista más auténtícamente científico. En otras palabras, las nociones de Dahrendorf a veces son impresionistas y auto-encomiásticas, pero ofrece ideas teóricas y metodológicas útiles para el análisis y con cierto substrato ideológico. En algún sentido, la explicación literaria del papel del intelectual que hace Dahtendorf, muestra que le interesa más estudiar las ataduras políticas del intelectual, que su tipo social. De ahí se sigue que Dahrendorf, mediante su modelo de grupos y élites, y a través de sus explicaciones de la autoridad y la legitimidad, ofrece una base para entender el papel del intelectual.

De la lectura de la obra de Dahrendorf podemos inferir algunas sugerencias: La inteliigentsia tiene un papel importante y tiene influencia sobre algunas de las condiciones técnicas que hacen posible que los quasi-grupos se conviertan en grupos de interés. Este papel es desempeñado junto con los procesos del liderazgo político. $\mathrm{Ni}$ los intelectuales ni los líderes de una élite dominante son factores determinantes sino más bien «pre-requisitos técnicos» (1959: 185). En las a.i.c. la aparición de grupos de interés presupone ciertas «condiciones técnicas» como por ejemplo élites dirigentes, miembros, estatutos y personal. Concretamente, la función del estatuto es codificiar la ideologia, weltanschauungs, valores y objetivos concretos de 
los grupos de interés, «racionalizar» la organización de los intereses manifiestos de estos grupos. Dahrendorf sigue aquí la idea de estatuto (o "valores») de Malinowski. ${ }^{18}$

¿Cómo elabora ideológicamente un quasi-grupo sus intereses latentes para producir intereses manifiestos? ¿La creación y articulación de un estatuto no es un proceso automático. Dahrendorf se enfrenta al problema de la articulación social de la ideologia. Y resuelve este problema subrayando la función del intelectual y la de los líderes políticos. Siempre bay una persona (ideólogo) o una camarilla que asume la tarea de articular una ideología (estatuto) o de incorporar un sistema de ideas que servirá como programa a los grupos de interés. Dahrendorf se refiere aquí a los ejemplos de Marx (como científico social, ideólogo y organizador del movimiento socialista), de las tesis de Weber sobre el papel de los intelectuales religiosos en saciedades tradicionales no capitalistas, y el del Calvinismo en el capitalismo inglés.

En resumen, desde el punto de vista de la articulación racional de los grupos de interés, los intelectuales son quienes hacen la ideología (Dahrendorf 1959: 186; 1967: 284). Desde el punto de vista de los grupos y los conflictos, los intelectuales no "crean» el conflicto sino que, más bien. son sobre todo una "variable participante». Son, para usar la nueva metáfora de Dahrendorf, «los tocólogos de los grupos conflictivos» (1959: 168).

Dahrendorf sugiere otras explicaciones para el entendimiento completo del papel del intelectual, en relación al problema de la legitimidad en las relaciones de autoridad. Hemos visto cómo Dabrendorf considera que en las a.i.c. hay siempre conflicto de intereses. Y para él, conflicto de intereses equivale a conflicto de legitimidades. En el conflicto entre clases hay siem. pre un grupo de interés que defiende la legitimidad establecida y, por otro lado, un grupo emergente que presenta una anti-legitimidad. Esto demuestra la «ilegitimidad potencial de todas las relaciones de autoridad» (1959: 176).

Si ocurre ésto, ¿cuáles son las relaciones entre los papeles del intelectual y el conflicto de legitimidades (en el conflicto entre clases)? Parece que Dahrendorf sugiere que si se puede decir que el intelectual es un creador de ideologías y es también un factor que interviene en el conflicto, se podrá asimismo afirmar que es un productor de legitimidad o bien un «demoledor» de la ilegitimidad o portador de una anti-legitimidad:

18. Malinowski define un «sistema de valores para cuya consecución los seres humanos se organzzan» (1944: 52). 
En toda asociación, los intereses del grupo dominante son los valores que constituyen la ideología de la legitimidad de su domi. nio, mientras que los intereses del grupo sometido constituyen una amenaza contra esta ideología y las relaciones sociales que cobija (Dahrendorf, 1959: 176).

Anteriormente habiamos visto que Dahrendorf critica la explicación unidimensional del intelectual, fuera ésta culturalista o profesional. Por otro lado, se muestra tajante a la hora de situar a los intelectuales en el terreno político. Siguiendo estas ideas, Dahrendorf analiza la noción dic] intelectual como alguien caracterizado «sobre todo por su uso independiente y deliberado de la palabra» (1967: 281). Aclara que tal declaración supone que la palabra en manos de los intelectuales es un medio de expresat convicciones y explicaciones más que un simple medio de comunicación. Es decir, que la palabra puede ser un arma política (1967: 281). Es cierto que, a veces, el uso de la palabra implica un cierto grado de disranciación. Sin embargo, esta distancia es la excepción y no la regla. Dahrendorf nota aquí que distancia e inmediatez, habiar y actuar, pueden considerarse como dos caras de la misma realidad. ¿Es cierto que quien habla no actúa ${ }^{19}$ Dahrendorf sugiere que quienes están en el poder tienen motivos especiales para no hablar, y que estos mismos motivos se encuentran en el caso de sus burocracias. Por otro lado, quienes disienten tienen dificultades para actuar. El papel del intelectual como aquél que puede expresar sus convicciones y dar explicaciones tanto como crítico como en favor de la legitimidad se basa en un tipo especial de distancia, que existe tanto para los intelectuales conservadores como para los izquierdistas; así «es la condición de la distancia y no un status con privilegios garantizados lo que libra a la gente de la prisión del poder y la dominación» (Dabrendorf, 1967: 282). Todos estos pensamientos le llevan a una conclusión: que los intelectuales no viven en el Olimpo de la palabra ní en un mundo de formas simbólicas, sino que simbolizan la estructura de su sociedad. Aquí Dahrendorf se refiere a la posición de distancia-inmediatez que caracteriza a los intelectuales.

Al analizar a los intelectuales alemanes, Dahrendorf encuentra las siguientes actitudes: a) «clásica»; b) «romántica»; c) «trágica»; y d) «crítica». Cada tipo podría ser representado por un intelectual de importancia como

19. Gramsci (1970) ha dado respuestas concretas a esta pregunta al analizar el papel del intelectual "orgánico» y el del "tradicional». Pueden encontrarse respuestas contemporáneas en las obras de Mills (1953), Shils (1972), Kadushin (1973), Careaga (1972), Lipset (1960), Hofstander (1966) y Marsal (1971) entre otros. 
Goethe, Hegel, Marx o Marcuse. Es más, cada actitud significa que se recalca más un aspecto u otro de la orientación distancia-inmediatez, y objetivos tales como ideología, búsqueda de la verdad, búsqueda de la libertad y compromiso moral, y liberación de la «topología» de los estereotipos. Parece que Dahrendorf explica el proceso de evolución de los intelectuales, de su actitud "clásica» a la "crítica», como un proceso que pasa de una sujeción al poder hacia una distancia y una crítica cada vez mayores ante ese poder. Si lo hemos entendido bien, lo que Dahrendorf trats de decir es que en la historia alemana, los intelectuales se ban ido desplazando de posiciones derechistas a posiciones izquierdistas. Dahrendorf llega a la misma conclusión que Karl Manheim en su Ideología y utopia (1936: $154-5,157$ ), a saber, que la evolución de las intelligentsias. en el seno dc las a.i.c., llega a «derivar libremente», la que para Dahrendorf significa que el intelectual queda «libre de los requisitos del papel familiar y del estrato social, de la iglesia y hasta incluso quizás de los de la ocupación. De este modo puede llegar a la "síntesis dinámica"... de distancia y pertenencia, alienación y participación, crítica y aceptación, que determina su bapel» (1967; 289).

\section{Canclusiones}

Ha habido muchos críticos que se han opuesto a las diversas obtas de Dahrendorf, ${ }^{20}$ pero, al parecer, no hay ningún análisis sistemático de toda su obra. Mayer (1960) considera a Dahrendorf como un teórico del conflicto que equilibra los puntos de vista unilaterales de la teoría funcional; para Coser (1962) las explicaciones de Dahrendorf están en el contexto de una sociología de la autoridad más en el de una teoría de las clases sociales; Van den Berge recalca el cauteloso determinismo de Dahrendorf y su fracaso en demostrar por qué la autoridad es previa a las relaciones de propiedad en Marx; ${ }^{21}$ y para Werlin (1968) el análisis que Dahrendorf hace de!

20. Tendríamos que mencionar aqui: Berget (1970), Coser (1960; 1962; 1968), Francis (1959), Lopreato (1967; 281), Mayer (1958; 1960), North (1968), Ruciman (1968), Shils (1970: 799), Wertin (1968), Wolff (1962).

21. Vet Van der Berghe, "Dialéctica y Funcionalismo: hacia una sintesis teórica" (1963: 700-701). 
conflicto de clase no llega a dar un análisis histótico satisfactorio. ${ }^{22}$ Quizás la única excepción a la regla sea Weingart $(1969){ }^{23}$ Su exhaustiva crítica se basa en la consideración de Dahrendorf como un neofuncionalista. Weingart comenta que Dahtendorf trata de it más allá de Parsons construyendo un sistema conceptual que, de hecho, no hace más que reflejar «su naturaleza puramente funcionalista» (Weingart, 1969: 161-162). Además. para Weingart, las explicaciones de Dahrendorf relativas a la autoridad, el poder y el conflicto son presentadas en términos voluntaristas.

Respecto a los intelectuales, algunas de las hipótesis que hemos mostra. do más arriba son básicas para un análisis empírico del papel de los intelectuales como: a) productores de ideología; b) legitimadores de las élites dominantes en el proceso de emergencia (o crepúscula) de los grupos de interés; c) creadores de la conciencia social de los quasi-grupos; y d) participantes en el conflicto entre grupos y clases.

Sin embargo se pueden encontrar contradicciones. Si interpretamos co rrectamente a Dahrendorf, está de acuerdo con la visión de Marx do los tipos históricos de compromisos de los intelectuales, que son asimismo papeles revolucionarios: a) el intelectual burgués. b) el intelectual socialista utópico, y c) el intelectual comunista. ${ }^{24}$

En el tema de los intelectuales, Dahrendorf trata de llegar a unna síntesis semejante a la que busca con su teoría de la clase y el conflicto de clases: en el terreno del conflicto de clases trata de ir más lejos que Marx ${ }^{25}$ y Parsons, ${ }^{26}$ para poder construir una alternativa diferente. ${ }^{27}$ En lo que se refiere al intelectual trata de construir una síntesis entre Marx y Mannhcim, algo que es más un esfuerzo contradictorio que una salida auténtica.

Por lo que hace al análisis empírico de los intelectuales Dahrendorf termina con un modelo cercano a las ideas de la freiscbwebende intelligenz

22. Podría preguntarse, en relación a toda la obra de Dahrendorf si es posible ir más allá del a-histórico Parsons con este tipo de limitaciones.

23. Weingart ha construido la crítica quizás más sistemática de la síntesis realizada por Dahrendorf entre Marx y Parsons en «Beyond Parons?: A Critique of Ralf Dahrendorf's Conflict Theo " (1969) ("¿Más allá de Parsons?: crítica de la teoría del conflicto de Dahrendorf".

24. Dahrendorf (1959: 14, 17, 25). De hecho Dahrendort aplica lo sustancial de estos tipos a los cambios de la élite intelectual alemana (1967: 283-289).

25. Ver Dahrendorf (1959: 117-156).

26. Ver Dahrendorf (1956), (1958 a), (1958 b), (1967 a), (1968).

27. Ver «Hacia una teoría del conflicto social» (1958 a); Clase y conflicto de clases en la sociedad industrial (1959); Conflict after Class (1967 a) (Conflicto según la clase); y Essays on the Theory of Society (1968). (Ensayos sobre la teoria de la sociedad). 
de Mannheim, aunque utiliza al mismo tiempo conceptos de Weber y de Parsons. Sin embargo, no deberíamos olvidar que el punto de partida de Dahrendorf está muy cargado de conceptos marxianos sobre la articulación social de la ideología. Según nuestra opinión. Dahrendorf cae en el mismo tipo de reducciones que Mannheim.

Probablemente la idea más sugerente de Dahrendorf es la que se refiere al papel de los «bufones»-intelectuales. Para usar sus propias palabras, éstos constituyen el único grupo social capaz de «romper el círculo vicioso de las condiciones sociales y de realizar y efectuar nuevos comienzos» (1967: 295). Deberfamos preguntarnos si esta hipótesis no es simplemente creer en un espejismo que refleja más un compromiso personal de Dahrendorf que una definición eficaz para el análisis de los intelectuales. Si fuera así, Dahrendorf estaría también dentro, y no «fuera de la Utopía».

Obras mencionadas en el texto

(Book review) $=$ (Crítica de libro)

(Mimeograph copy from Prof. Kadushin's forthcoming book) $=$ (Copia a ciclostil del último libro del Prof. Kadushin, de próxima aparición).

(First ed., in German: 1852) = (Primera edición en alemán: 1852). 